\title{
Variaciones fonológicas que producen rupturas en el discurso hablado entre hablantes de inglés no-nativos: Implicancias pedagógicas
}

\author{
Pedro Luis LUCHINI \\ Universidad Nacional de Mar del Plata (Argentina) \\ Facultad de Humanidades \\ luchinipedroluis@gmail.com.ar
}

Recibido: junio 2010

Aceptado: marzo 2011

\section{RESUMEN}

Actualmente, el inglés es empleado como segunda lengua en interacciones entre hablantes de inglés no-nativos provenientes de diferentes nacionalidades. El propósito de este trabajo es identificar variaciones fonológicas que producen rupturas en el discurso hablado en interacciones entre ellos. Utilizando algunas de las características fonológicas que conforman la "Lingua Franca Core" propuesta por Jenkins (2000), estas desviaciones fueron clasificadas conforme a su impacto en la preservación de la mutua inteligibilidad. Participaron en este estudio catorce estudiantes internacionales de la Universidad de Concordia, en Montreal, Canadá $^{1}$. Estos alumnos fueron agrupados en pares para completar una tarea comunicativa en inglés, usando sus propios acentos regionales. Posteriormente, el entrevistador, junto con cada par, analizó cada una de las instancias en donde la comunicación había sido interrumpida. La tarea y la posterior entrevista fueron grabadas, transcriptas y analizadas. Los resultados demostraron que las desviaciones segmentales, conjuntamente con la incorrecta localización del acento tonal resultaron ser los principales factores que afectaron la inteligibilidad entre los interlocutores. Finalmente, se abordan algunas cuestiones relacionadas con las implicancias pedagógicas del estudio.

Palabras clave: inglés como lengua internacional- inteligibilidad- fonología aplicada.

${ }^{1}$ Este proyecto de investigación fue realizado en el marco de la beca perteneciente al Programa de Perfeccionamiento docente, FEP (Faculty Enrichment Program), otorgada en 2007 al autor de este trabajo por medio de la Asociación Argentina de Estudios Canadienses, ASAEC en conjunto con el Ministerio de Relaciones Internacionales, Embajada de Canadá en Buenos Aires, Argentina. 


\title{
Phonological variation that cause communication breakdown among speaking of no-native English. Pedagogic Implications
}

\begin{abstract}
At present, English is being used as a second language in interactions among English native speakers and non-native speakers from different nationalities. The spread of English often creates special challenges related to mutual intelligibility of non-native speakers whose first languages are different. The purpose of this paper is two-fold: (i) to identify elements of speech that contribute to unintelligibility between non-native speakers of different L1s, and (ii) to analyze the impact that an activity on English as an international language (EIL) had on 21 pre-service students -whose L1 was Spanish- attending a pronunciation course at Universidad Nacional de Mar del Plata, Argentina. The speech data for this study were collected from 14 international students studying at Concordia University, Montreal, Canada. The students, who came from various backgrounds were paired with a different-L1 partner and recorded in a dyadic interaction task. Each pair of students then participated in a stimulated recall task, listening to their recording and identifying instances of communication breakdown. These recordings were transcribed and analyzed by the 21 student teachers. Then, using the Lingua Franca Core as an analytical framework (Jenkins, 2000), they identified cases of communication breakdown for possible sources of unintelligibility. Results revealed that non-native production of some segments, especially when they are combined with misplacement of nuclear stress, were the greatest source of unintelligibility between differentL1 speakers. Most listeners acknowledged having gained some awareness of the importance of EIL for their own language development. Some pedagogical implications for the teaching of EIL were discussed.
\end{abstract}

Keywords: English as an international language - intelligibility - applied phonology.

\section{Variations phonologiques qui produisent des ruptures dans le discours parlé en anglais d'interlocuteurs non natifs d'anglais : implications pédagogiques}

\section{RÉSUMÉ}

Actuellement, l'anglais est employé comme deuxième langue dans des interactions entre des interlocuteurs non-natifs d'anglais provenant de différentes nationalités. Le but de ce travail est d'identifier les variations phonologiques qui produisent des ruptures dans le discours parlé dans des interactions entre eux. En utilisant certaines des caractéristiques phonologiques qui conforment la "Lingua Franca Core" proposée par Jenkins (2000), ces déviations ont été classées selon leur impact dans la préservation de l'intelligibilité mutuelle. Quatorze étudiants internationaux de l'Université de Concorde, à Montréal, au Canada, ont participé à cette étude. Ces élèves ont été groupés en paires pour compléter une tâche communicative en anglais, en utilisant leurs propres accents régionaux. Par la suite, avec chaque paire, l'interviewer a analysé chacune des instances où la communication avait été interrompue. La tâche et l'interview postérieures ont été enregistrées, transcrites et analysées. Les résultats ont démontré que les déviations segmentales, ensemble avec la localisation incorrecte de l'accent tonal ont été les facteurs principaux qui ont affecté l'intelligibilité entre les interlocuteurs. Finalement, on aborde quelques questions relatives aux implications pédagogiques de l'étude.

Mots-clés : l'anglais comme langue internationale, intelligibilité, phonologique appliquée. 
SUMARIO: 1. Marco teórico. 2. Método. 2.1. Contexto y participantes. 2.2. Instrumentos y procedimiento. 3. Análisis. 3.1. Sustitución de sonidos. 3.2. Omisión de una consonante. 3.3. Adición de sonidos: epéntesis y paragoge. 3.4. Duración de fonemas vocálicos. 3.5. Preservación del fonema 3:. 3.6. Localización del acento tonal combinado con desviaciones sonoras. 3.7. Errores a niveles sintáctico y semántico. 4. Implicancias pedagógicas. 5. Discusión y conclusión final. Referencias bibliográficas. Apéndice.

\section{MARCO TEORICO}

Hoy en día, unos pocos pueden disputar el hecho de que el inglés es la lengua franca por excelencia utilizada entre hablantes no nativos, provenientes de diferentes nacionalidades alrededor del mundo. Sin embargo, como cualquier otro idioma, éste esta compuesto por una diversa gama de variedades. El inglés estándar, el que comúnmente se enseña en colegios, escuelas y universidades en muchos contextos, es el inglés escrito. En cuanto al inglés hablado, sin embargo, la primera pregunta que surge es ¿qué tipo de acento se debe enseñar y aprender? A la fecha, la elección de muchos profesionales en el área de pronunciación ha consistido en el acento británico denominado Received Pronunciation ( $R P)$ y/o el acento americano llamado General American (GA). Sin embargo, Jennifer Jenkins (2000), en su libro "The Phonology of English as an International Language" propone una tercera opción a la que llama Lingua Franca Core ( $L F C$ ) para ser utilizada por hablantes no-nativos de inglés que no desean o que no pueden aproximarse a las normas impuestas por los modelos estándares dominantes anteriormente mencionados.

El trabajo de esta investigadora está fuertemente influenciado por el movimiento World Englishes o English as an International Language (El Inglés como Lengua Internacional) (Dauer, 2005). Este movimiento ha revelado que, a la fecha, el inglés es utilizado como una herramienta para la comunicación entre hablantes nativos/nativos, nativos/no-nativos y aún más entre no-nativos. Teniendo en cuenta que más de 350 millones de anglo-parlantes no-nativos habitan el mundo, la expansión global del inglés, durante los últimos años, ha hecho que se transformara en la lengua franca o lengua internacional por antonomasia en todos los ámbitos: comercial, educativo, tecnológico e investigativo, entre otros (Brown, 2001).

Con el objetivo de lograr que estos hablantes no-nativos de inglés se puedan comunicar efectivamente entre sí, la inteligibilidad mutua debe ser garantizada. El principio de inteligibilidad fonológica, según Levis (2005), implica que un hablante que es inteligible en una segunda lengua (L2) debe ser fácil de comprender. Además, este principio sostiene que (1) la comunicación puede ser exitosa aún cuando los L2 hablantes preserven sus propios acentos locales o regionales, (2) no hay una correlación directa entre estos acentos regionales y su comprensión, y (3) ciertos errores de pronunciación pueden tener un rol desproporcionado en el impedimento de la comprensión. El principio de inteligibilidad implica, además, que diferentes rasgos o características fonológicas pueden producir diferentes efectos en la comprensión auditiva. Por lo tanto, Levis sugiere la incorporación de estas características en la enseñaza de la pronunciación del inglés como lengua internacional. Por otro lado, en 
su trabajo, Jenkins (2000) identifica una serie de características y rasgos fonológicos que todos los hablantes de inglés de diferentes nacionalidades comparten, y que considera indispensables para lograr una comunicación efectiva y eficiente.

Durante varios años, se ha argumentado, y aún se sigue haciéndolo en varios contextos nacionales e internacionales, que la fonología segmental tiene un efecto mucho menos perjudicial sobre el logro de la inteligibilidad que los rasgos suprasegmentales de la lengua. Sin embargo, Jenkins (2000), entre otros investigadores en el área, afirma que, a la fecha, se han podido registrar muy pocos resultados provenientes de investigaciones empíricas que han dado cuenta sobre esta temática. En sus estudios, esta investigadora propone un listado de rasgos y características fonológicas que, según sus resultados, son esenciales para preservar la inteligibilidad en contextos internacionales. Basándome en estos datos, en mi propia experiencia como hablante no-nativo del inglés y en el resultado de mis propias investigaciones (Luchini 2007a, 2007b, Luchini y Roselló 2007, Luchini 2006, Luchini 2005a, 2005b, 2005c, Luchini 2004), en este estudio se explorarán aquellos aspectos fonológicos que se consideran primordiales para lograr inteligibilidad fonológica en contextos internacionales:

1. Sustitución de sonidos debido a problemas fonéticos y dificultades articulatorias en la realización de los sonidos meta. Por ejemplo, la sustitución de la consonante sorda, africada / $\mathrm{tt} \int /$ por la sorda, post-alveolar, fricativa $/ S /$ en el vocablo "beach" da como resultado /'bi: $\int /$ en lugar de /'bi:ts/.

2. Omisión de una consonante o de un grupo consonántico. Por ejemplo, la palabra "construction" /Kəns'tr $\Lambda \mathrm{k} \int ə \mathrm{n} /$, puede sufrir la omisión de uno o dos grupos consonánticos: /str/ y $/ \mathrm{kS} /$ en posición media, y ser, a la vez, remplazados por la consonante sorda, fricativa, dorsoprepalatal $/ \mathrm{S} /$. Siguiendo este fenómeno fonológico, el resultado de esta palabra, sobre todo en contextos asiáticos, puede ser $/ \mathrm{K} ə n^{\prime} \int \Lambda \mathrm{n} \int ə \mathrm{n} /$.

3. Adición de sonidos (epéntesis y paragoge):

Epéntesis es la adición de un fonema, en general vocálico, en el interior de una secuencia fonológica. Por ejemplo: 'hamster' comunmente pronunciada con un sonido /p/, /'hæmpstər/.

Paragoge es la adición de un fonema o secuencia de segmentos, por lo general vocálicos, al final de una palabra. Por ejemplo: 'college' /'kolıdzi/, 'first'/'fз:stə/, 'last'/'la:stə/.

4. Distinciones en duración de vocales (cantidad): Las vocales tensas son relativamente más largas en duración que las laxas. Por ejemplo, la vocal tensa /a:/, como en 'farm' /'fa:m/ es más larga en duración que la vocal laxa /i/ en ' $\mathrm{dim}$ ' $/ \mathrm{dim} / \mathrm{en}$ un mismo contexto. Esto se debe a que las vocales tensas son alofónicamente más largas que las laxas. Se denomina "duración inherente" a la duración de las vocales independientemente del contexto en el 
que aparezcan. La "duración contextual" (Rogers, 2000:78), por otro lado, se refiere a la duración de las vocales en un contexto determinado, por ejemplo, delante de consonantes. Cualquier vocal delante de una consonante sorda en posición final, se debe acortar, mientras que la duración de una vocal frente a una consonante sonora en la misma posición, se debe mantener (Rogers, 2000). Por ejemplo, las vocales en la columna izquierda tienen una duración más corta que las que se encuentran a la derecha:

$$
\begin{array}{ll}
\text { feet /'fi:t/ } & - \text { feed /'fi:d/ } \\
\text { cart /'ka:t/ } & - \text { card /'ka:d/ } \\
\text { heard /'hs:d/ } & - \text { hurt /'hs:t/ }
\end{array}
$$

5. La única vocal que debe ser preservada con respecto a su calidad y cantidad debe ser la $/ \mathbf{3}: /$, ya que su sustitución por otro fonema -frecuentemente /a:/generalmente, atenta contra la inteligibilidad. Por ejemplo, en el caso de la palabra "third" $/{ }^{\prime} \theta \cdot 3: \mathrm{d} /$, los hablantes asiáticos comúnmente remplazan el fonema /3:/ por la vocal /a:/, pronunciando este vocablo como /' $\theta \mathrm{a}: \mathrm{d} /$.

6. Localización y producción del acento tonal: Al acento tonal se lo conoce también como prominencia tónica. El elemento que contiene esta prominencia es el elemento tónico, pie tónico, o sílaba tónica. Halliday (1985), en su enfoque sistémico funcional, denomina a este elemento "information focus", por acarrear el foco de la información. La localización errónea de este acento tonal o prominencia tónica, según Jenkins (2000), en conjunto con desviaciones segmentales, resulta ser una combinación devastadora para el logro de la inteligibilidad, ya que esta composición generalmente coincide con la localización del foco de la información (García Jurado y Arenas, 2005). En línea con esto, por ejemplo, la palabra "priority" / /prar'oriti/, puede ser realizada por algunos hablantes asiáticos como /'praiorti:/.

Siguiendo este marco teórico, en este trabajo se analizó e interpretó una serie de grabaciones llevadas a cabo entre hablantes no-nativos de inglés, originarios de diferentes países. Estos participantes fueron agrupados en parejas para realizar una tarea comunicativa en inglés. Inmediatamente después de esta tarea, se entrevistó a cada par con el objetivo de analizar aquellas instancias en donde la comunicación entre los hablantes había sido obstaculizada debido a variaciones fonológicas. Dichas actividades (tarea y conversación post-tarea) fueron grabadas y transcriptas para luego ser analizadas e interpretadas. 


\section{METODO}

\subsection{Contexto y participantes}

Los participantes en este trabajo fueron catorce estudiantes que, al momento de realizar las entrevistas (septiembre, 2007) estaban inscriptos en la Universidad de Concordia, Montreal, Canadá. Estos alumnos son originarios de los siguientes países: China, Vietnam, Corea del Sur, Jordania, Perú, Ucrania, Honduras y Bangladesh. Como requisito para poder ingresar a esta universidad, los estudiantes internacionales deben acreditar un nivel de inglés equivalente al TOEFL (ETS Organization). En caso de no contar con este nivel de competencia lingüística en L2, deben tomar un curso preparatorio a tal fin que lo ofrece esta misma universidad. La edad promedio de estos alumnos al realizar las entrevistas fue de 20.98 (18-26) y su nivel de inglés promedio fue intermedio/avanzado.

\subsection{Instrumentos y Procedimiento}

Los instrumentos utilizados para la recolección de datos fueron una tarea comunicativa (Apéndice A), extraída de un examen diseñado para evaluar la pronunciación en la voz hablada espontánea (Luchini, 2004), y una entrevista posttarea en la cual se analizaron, con cada grupo de pares, aquellos factores fonológicos que, durante la concreción de esta tarea, obstaculizaron la comunicación. La tarea comunicativa consistió en presentar a los alumnos con una situación imaginaria en la cual, luego de haber ganado mucho dinero, se les solicitaba que eligieran tres de cinco ítems mostrados en una serie de fotos (un avión, una casa en la playa, un automóvil, un laboratorio para la investigación científica y gente donando dinero a los más pobres). Cada pareja contó con 60 minutos para mirar las fotos, y 3 minutos para concretar la actividad. En el caso de haber existido rupturas en la comunicación durante el desarrollo de la actividad, éstas fueron analizadas en una conversación post-tarea llevada a cabo entre la pareja de alumnos y el entrevistador. Tanto la tarea como esta última conversación fueron grabadas y transcriptas por dos especialistas en fonología por separado para su posterior evaluación. En aquellos casos en que se detectaron divergencias entre los especialistas en el material de habla transcripto, se acordó en reverlos en conjunto para determinar la versión correcta.

\section{ANALISIS}

En los 54 minutos totales de grabación (tareas y conversaciones post-tarea) se detectaron 32 instancias en donde el discurso hablado espontáneo fue interrumpido entre los hablantes, primordialmente, debido a desviaciones fonológicas. De las 32 instancias analizadas, por razones de practicidad y espacio, solo 10 de éstas fueron seleccionadas en este trabajo con el objetivo de ilustrar -desde una perspectiva fonológica- qué sucede cuando hablantes no-nativos de inglés de diferentes 
nacionalidades, interactúan entre sí (las grabaciones están disponibles). En los fragmentos seleccionados que se muestran a continuación, las palabras en donde se registraron desviaciones fonológicas se presentan en negrita y sus transcripciones fonéticas debajo.

\subsection{Sustitución de sonidos}

Países de origen: Vietnam - Ucrania

Vietnam: 4 años de residencia en Montreal. Ingresante a la universidad. Edad: 19.

Ucrania: 6 años de residencia en Montreal, alumna de postgrado en lingüística aplicada en Concordia. Edad: 26.

China: Because I love beach.

Ucrania: You love beach.

China: Yeah, my hometown has a, erm, beautiful beach.

Ucrania: Ah..., sorry?

/'bi:S/

China: Erm..., my hometown has a beautiful beach. Nice ocean and sand.

Ucrania: Mmm...

China:Yeah...

En este primer caso, la desviación fonológica ocurrió cuando la hablante vietnamita sustituyó la consonante sorda, africada /t $\mathrm{f} /$ por la sorda, post-alveolar, fricativa / $/$ / en el último ítem léxico ("beach") de la frase entonativa, donde el foco de la información nueva reside. Si bien el acento tonal en esta palabra se encuentra localizado en el lugar correcto, esta instancia demuestra que la sustitución de un fonema por otro en una palabra cargada de significado y contenido, como lo es este vocablo en este caso que lleva consigo información nueva, resulta ser devastadora en la comunicación entre dos hablantes no-nativos de inglés.

Ucrania: Ok, so wha... what is left you would give to the poor.

China: Yeah... (...) Mmm, erm, because I..., I want..., I want to help the more

/'hev/

poorly...

/'pa:li/

May... mmm...

Ucrania: You want..., sorry, you want what?

China: Help them ... live better.

Ucrania: Ok...

En esta segunda instancia, la hablante vietnamita reemplazó el grupo consonántico /lp/ en la palabra "help" /'help/ por el fonema /v/, realizándola como /'hev/. Por otro lado, en la misma frase entonativa, esta alumna sustituyó en la palabra "poorly" /'po:li/, la vocal larga, posterior, semi-cerrada / $\mathrm{s}:$ / por la larga, posterior, abierta /ai/, 
resultando /'pa:li/ . Estos dos casos de sustitución, en dos palabras diferentes dentro de una misma unidad de información impidieron la comprensión por parte de la participante ucraniana que, a pesar de estar equipada con mayores recursos lingüísticos que su compañera, dada su mayor formación académica y tiempo de estadía en Montreal, fue incapaz de descifrar la desviación fonológica. Una vez más, los datos evidencian que son las desviaciones fonológicas a nivel segmental las que resultan ser devastadoras para el logro de la mutual inteligibilidad entre hablantes nonativos de inglés, provenientes de diferentes nacionalidades.

\subsection{Omisión de una consonante o grupo consonántico}

Países de origen: China - Bangladesh

China: 2 años de residencia en Montreal. Estudiante de biología en la universidad. Edad: 22.

Bangladesh: 8 meses de residencia en Montreal, alumno de sociología. Edad: 26.

Bangladesh: And... if I want to help poor people is more difficult... is... for me... because almost sixty per cent people are poor. But, I...

/'sristi/

China: Si... Si...

Bangladesh: Sixty per cent.

China: Sixty per cent!

En este fragmento, la ruptura en la comunicación fue provocada por dos desviaciones fonológicas en la palabra "sixty", /'sıksti/. Por un lado, el hablante de Bangladesh omitió la consonante $/ \mathrm{k} /$ en el grupo consonántico $/ \mathrm{sk} /$, por lo tanto, pronunció esta palabra como /'sisti/. Generalmente, la mayoría de los hablantes asiáticos encuentran algún tipo de dificultad fonética y/o articulatoria en la producción de algunos grupos consonánticos. Ante esta dificultad, muchos de estos hablantes deciden omitirlos totalmente o parcialmente, como bien pudo haber sido la reacción de este participante en este caso. Por otro lado, en esta misma palabra, este participante reemplazó una vocal corta /I/ por una larga /i:/, realizando entonces la palabra "sixty" como /'sissti/. Como Jenkins (2000) explica en su teoría, los estudiantes de inglés raras veces acortan las vocales que preceden una consonante sorda, hecho que causa una desviación en la duración y calidad de esta vocal, en la conversación post-tarea, la participante china explicó que el factor que la confundió en este caso, fue principalmente la omisión de la consonante /k/ en el grupo /sk/.

Países de origen: Vietnam - China

Vietnam: 4 años en Montreal. Ingresante a la universidad. Edad: 22.

China: 2 años en Montreal. Estudiante de negocios, primer año. Edad: 23 
Vietnam: And, okay, let' see. If you have a lot of money, what are you gonna buy?

China: Yes, well, I have a car.

Vietnam: Me too, I want a car too.

China: Yes, a car, and... I don't... I don't understand. What is it?

Vietnam: This looks like the house, construction.

China: Construction?

$$
/ \mathrm{k} ə \mathrm{n} / \Lambda \mathrm{n} \int \mathrm{n} /
$$

Vietnam: Like building houses.

China: So, You'd use the money to build houses but what is this?

En esta fragmento se presenta una ruptura con la palabra "construction" /Kəns'tr $\Lambda \mathrm{k} \int ə n /$, en la cual la hablante vietnamita omite dos grupos consonánticos: /str/ y $/ \mathrm{kS} /$ en posición media, y los remplaza por la consonante sorda, fricativa, dorsoprepalatal $/ \mathrm{S} /$, produciéndola como /Kən' $\int \Lambda \mathrm{n} \int ə \mathrm{n} /$. Independientemente de este caso, con esta pareja de alumnos casi no se registraron casos de rupturas en su conversación, producto de desviaciones fonológicas. En la conversación post-tarea, los interlocutores reconocieron y explicaron que, si bien sus idiomas de origen son diferentes, aparentemente, no les resulta difícil interactuar con otros hablantes asiáticos ya que pareciera ser que comparten algunos aspectos fonológicos en común como así otros tantos para-lingüísticos que, evidentemente, contribuyen al logro de una efectiva comunicación en L2. Con respecto a este tema, la participante vietnamita dijo:

Yeah, well, I don't know if we have the same problem but it's easy for us to understand each other...but if you match me with another people such as like...Arabic...eh...People who speaks Spanish...I have hard time to understand people who speaks Spanish...because they speak like "Yeee" and they don't break, break...between two word...so it's as if you hear the word stick together..

Traducción: Bueno, no se si tenemos los mismos problemas, pero es fácil para nosotros entendernos mutuamente... pero si me agrupa con otra persona como por ejemplo... un árabe... eh....la gente que habla español... Tengo problemas para entenderle a las personas que hablan español... porque hablan como "ye ye ye" y no paran, no paran entre palabra y palabra.... Entonces, es como si escucharas las palabras pegadas, juntas...

En línea con lo expuesto por esta participante, el resto de las parejas en este estudio fueron agrupadas teniendo en cuenta su sugerencia. Por cierto, esta misma alumna se ofreció a replicar esta misma actividad con otro participante - no asiáticopara poder identificar y estudiar las dificultades fonológicas que, según ella, muy a menudo, conllevan a obstruir la mutua inteligibilidad entre hablantes no-nativos de inglés (ver próximo caso: Perú/Vietnam). 


\subsection{Adicción de sonidos: epéntesis y paragoge}

Países de origen: Perú - Vietnam

Perú: 1 año de residencia en Montreal. Ingresante a la universidad. Edad: 21.

Vietnam: 4 años de residencia en Montreal. Ingresante a la universidad. Edad: 22.

Perú: ... friends...or... something ...

Vietnam: ... what kind of car do you want to buyed?

/'baid/

Perú: Mm... I don't know yet but...for sure a nice car. And you?

Vietnam: Er....Me? Er...I would buy sport car...er... Where do you want to go if you had a lot of money?

Países de origen: Vietnam - Ucrania

Vietnam: 4 años de residencia en Montreal. Ingresante a la universidad. Edad: 19.

Ucrania: 6 años de residencia en Montreal, alumna de postgrado en lingüística aplicada en Concordia. Edad: 26.

Vietnam: My name is Truc, I'm from Vietnam

Ucrania: Ok, hello, nice to meet you, my name is Tamara, I'm from Ukraine.

Vietnam: yeah, and, er, I'm going to choose the first is car.

Ucrania: $m$ h...

/'ka:rə/

Vietnam: yeah, er, because I want to, em, go to many place is more convenient.

Ucrania: Ok, so it's more convenient to travel by car. Good.

Países de origen: China - Bangladesh

China: 2 años de residencia en Montreal. Estudiante de biología en la universidad. Edad: 22.

Bangladesh: 8 meses de residencia en Montreal, alumno de sociología. Edad: 26.

China: Oh, Ok, sorry... What is your ... could you repeat your sentence?

Bangladesh: Oh! If I...

China: Your first... the last sentence.

/'f3:stə/ / 'la:stə/

Bangladesh: Oh... last sentence... er ... the first priority...

A pesar de que en algunos casos este fenómeno fonológico de adición de sonidos puede obstruir la inteligibilidad en contextos en donde el inglés es usado como lengua internacional, los resultados en este estudio demostraron que tanto la epéntesis con schwa /a/, como el fenómeno de paragoge no obstaculizaron la comunicación. Por cierto, es el fenómeno de la omisión de sonidos (consonantes o grupos consonánticos en posición inicial principalmente) que combinado con la incorrecta localización del 
acento tonal, se torna altamente amenazante para el logro de la mutua inteligibilidad entre hablantes no-nativos de inglés.

Con respecto a estos fenómenos, Jenkins (2000) postula que la adición de sonidos es menos factible que comprometa la inteligibilidad que la omisión de los mismos, ya que la forma subyacente es más fácil de recuperar. De hecho, paragoge, por ejemplo, en el caso de "college" /'kolidz/, en donde los hablantes de mandarín suelen agregar la vocal/i/ en posición final, puede servir para clarificar y asegurar la permanencia de la consonante sonora africada / $\mathrm{d} 3 /$ que la antecede. Para estos hablantes, por ejemplo, las reglas de paragoge son más difíciles de adquirir que las mismas reglas de omisión de consonantes, ya que éstos deben incorporar junto al fenómeno de paragoge, es decir la adición de un fonema al final de un vocablo, las consonantes oclusivas localizadas en posición final las cuales no existen en su L1. La omisión del fonema vocálico que se suma a una consonante oclusiva en posición final, en el caso de paragoge, ocasionaría la pérdida no solamente de esta vocal sino también de la misma consonante a la cual ésta se adosa. Por lo tanto, la palabra "college", por ejemplo, sería realizada como /'koli/. Si éste fuera el caso, entonces, no se podría distinguir la diferencia entre vocablos tales como "college", "colic", o "colleague", entre otros. Con respecto a este tema, en su estudio, Jenkins (2000) sugiere que en estos casos, el fenómeno de paragoge, al contrario que la omisión de fonemas, contribuye a aumentar la inteligibilidad en lugar de comprometerla, sobre todo en el caso de L2 oyentes con poca fluidez.

\subsection{Duración de fonemas vocálicos}

En este trabajo no se detectaron interrupciones en la comunicación entre los participantes debido a la incorrecta duración de los segmentos del habla exclusivamente. La mayoría de las rupturas en la comunicación se debieron a problemas de sustitución y omisión de sonidos, en algunos casos, combinados con la errónea localización del acento tonal.

\subsection{Preservación del fonema /3:/}

Países de origen: China - Bangladesh

China: 2 años de residencia en Montreal. Estudiante de biología en la universidad. Edad: 22.

Bangladesh: 8 meses de residencia en Montreal, alumno de sociología. Edad: 26.

Bangladesh: Uh... huh.

China: Yeah.

Bangladesh: All right. I said I'll buy a house and car, and my third thing...

China: Your what?

/'tard/

Bangladesh: My third thing. My last choice... 
China: Ah, ok...

Bangladesh: ... is the vacation. Vacation means, you already said this... I want to go... er... Hawaii.

Si bien en este caso, el participante de Bangladesh sustituyó la consonante inicial sorda fricativa dental $/ \theta /$ por la sorda oclusiva /t/ en la palabra "third", realizándola como /'ta:rd/, ésta no fue la verdadera causa de la ruptura en la comunicación. En la conversación post-tarea la hablante china no se refirió al fenómeno de sustitución de estas consonantes, sino a la desviación en la cantidad y calidad de la vocal /3:/ como el factor determinante que causó el quiebre en la interacción. Como lo anticipara Jenkins (2000) oportunamente en sus estudios, la distorsión en la calidad y/o cantidad de esta vocal, y su posterior substitución por otra -generalmente /a:/- ocasiona, a menudo, importantes rupturas en la comunicación.

\subsection{Localización del acento tonal combinado con desviaciones sonoras}

Países de origen: China - Bangladesh

China: 2 años de residencia en Montreal. Estudiante de biología en la universidad. Edad: 22.

Bangladesh: 8 meses de residencia en Montreal, alumno de sociología. Edad: 22.

Bangladesh.: ...shelter is the first priority to a man my life.

/'prairrti:/

China.: Oh, Ok, sorry... What is your ... could you repeat your sentence?

Bangladesh.: Oh! If I...

China.: Your first... the last sentence.

Bangladesh.: Oh... last sentence... er ... the first priority...

China.: Ok.

Bangladesh.: The first priority...

China.: What did you say there?

En este caso, el hablante de Bangladesh alteró la localización del acento tonal en la palabra "priority"/prai'oriti/, realizándola como/'praıərti:/. Es decir, realzó la primera silaba de esta palabra en lugar de hacerlo en la segunda. Este fenómeno prosódico trajo aparejado una serie de desviaciones fonológicas a nivel segmental. Por un lado, la sílaba prominente /'ori/ fue omitida y reemplazada por/Iər/. Por otro lado, la vocal débil /i/, en posición final, fue remplazada por la vocal larga /i:/, resultando entonces/'praiərti:/. En la entrevista post-tarea, los participantes acordaron que la combinación de la incorrecta localización del acento tonal junto a esta desviación sonora resultó ser un efecto devastador para el logro de la mutua inteligibilidad entre estos dos hablantes.

A continuación, se muestran dos ventanas acústicas a través de las cuales se compara la representación acústica de la palabra "priority" en forma de onda de energía. La primera realización fue producida por un hablante nativo de inglés 
(ventana 1), mientras que la segunda fue producida por el hablante de Bangladesh (ventana 2). Las zonas más marcadas en cada onda indican mayor condensación de energía, es decir, la localización de sílabas prominentes, acentuadas o ambos casos en conjunto.

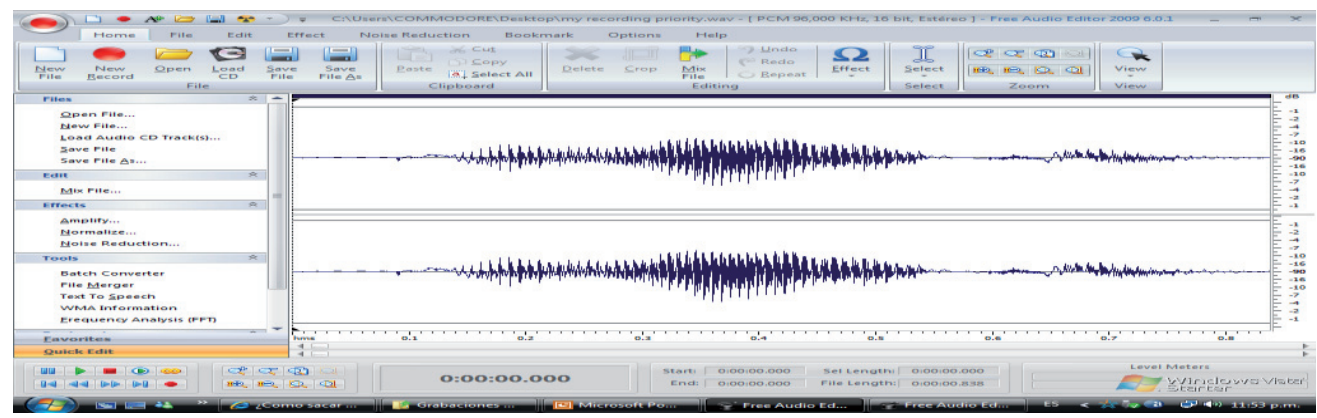

Ventana acústica 1- Localización del acento tonal en la sílaba correcta de la palabra "priority", realizada por el hablante nativo de inglés: /praI'JrIti/

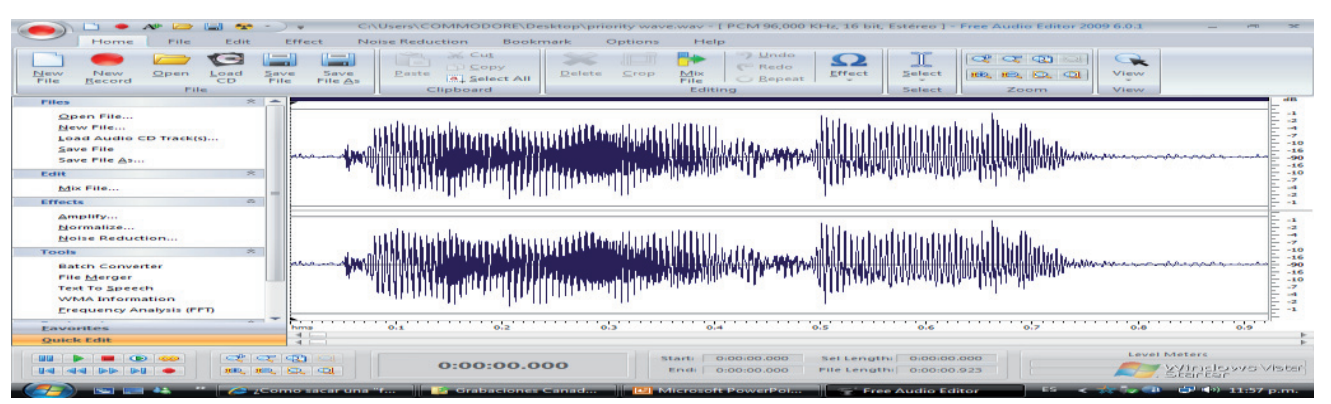

Ventana acústica 2- Localización del acento tonal en la sílaba incorrecta de la palabra "priority", realizada por el hablante de Bangladesh /'praırti:/.

Tal como se explicó anteriormente, en la ventana 2, donde la localización del acento tonal es incorrecta, se puede ver claramente la condensación de energía en la primera parte de la onda y un alargamiento hacia el final de la palabra, en donde el hablante de Bangladesh prolonga la duración de la vocal débil /i/, transformándola en /i:/.

Países de origen: Honduras - Corea del Sur

Honduras: 1 año de residencia en Montreal. Ingresante a la universidad. Edad: 21. Corea del Sur: 2 años de residencia en Montreal. Ingresante a la universidad. Edad: 22. 


\section{Honduras: A plane?}

Corea del Sur: Yeah, the plane, to...

Honduras: But planes are so expensive.

Corea del Sur: Oh, but you said if I had a lot of money it says (..?)

Honduras: Yeah, maybe.

Corea del Sur: And... I would have somebody to...ride... it for me.

Honduras: So, you'd also buy a pilot also.

$$
\text { /bar'lot/ }
$$

Corea del Sur: Buy what?

Honduras: A pilot?

$$
\text { /'bailot/ }
$$

Vietnam: Bye... a Bye lot?

$$
\text { /bai/ /a bai lot/ }
$$

\section{Oh, pilot. Just hire a pilot! Yeah!$$
\text { /'pailat/ /'pailat/ }
$$

En este fragmento, la ruptura en la comunicación se originó por dos desviaciones fonológicas producidas en el vocablo "pilot"/'pailət/. Por un lado, según lo conversado en la entrevista post-tarea, la hablante de Honduras, al no incluir la suficiente aspiración $/ \mathrm{h} /$ seguida de la consonante sorda oclusiva $/ \mathrm{p} /$ en posición inicial y en una sílaba acentuada, hizo que la oyente coreana la confundiera con la consonante sonora /b/. Sin la ayuda de esta aspiración, la oyente reconoció haber tenido dificultad en distinguir la sonoridad entre estos dos sonidos $/ \mathrm{p} / \mathrm{y} / \mathrm{b} /$.

De hecho, una consonante sorda en posición inicial y en una sílaba acentuada, producida sin su correspondiente aspiración puede ser fácilmente confundida por su contraparte sonora. La falta de aspiración en este contexto fonológico es muy común en L2 hablantes hispanos. Por ejemplo, el fonema /p/ no aspirado en posición inicial y en sílaba acentuada puede ser fácilmente confundido por $/ \mathrm{b} /, / \mathrm{t} /$ por $/ \mathrm{d} / \mathrm{y} / \mathrm{k} /$ por $/ \mathrm{g} /$ (Jenkins, 2000). Por otra parte, la participante de Honduras localizó el acento tonal en la última sílaba cuando debería haberlo hecho en la primera, realizando la palabra "pilot" como /baI'lot/. Los resultados obtenidos en este estudio, que se corresponden en gran medida con los presentados por Jenkins (2000) en su trabajo, indican que los mayores obstáculos fonológicos que impiden la mutua inteligibilidad entre hablantes no-nativos de inglés, originarios de diferentes países, parecen ser las desviaciones sonoras en combinación con la incorrecta localización o producción del acento tonal.

\subsection{Errores a niveles sintáctico y semántico}

Países de origen: Perú - Jordania

Perú: 1 año de estadía en Montreal. Ingresante a la universidad. Edad: 19.

Jordania: 2 años de estadía en Montreal. Ingresante a la universidad. Edad: 23.

Jordania: Well, the third thing I'm gonna do is gonna go for a vacation spend with a lot of money there, a lot of time, and then I'll think about other things. 
Perú: Yeah, yeah, of course. You can. You can enjoy your money. Well, with you, with your family or friends or something. And then you can help a lot of people, specially, if you don't know them. And, I don't know, and you will try to help deep closer friends, maybe they have some problems or something, and...

Jordania: Family's the first thing...

Perú: Yeah, of course family first, and then you can help people that you know like some poor people or something.

Si bien estos dos participantes cometieron errores a nivel sintáctico y semántico a lo largo del desarrollo de la tarea, no tuvieron rupturas significativas en la comunicación. En la conversación post-tarea, los participantes explicaron que la razón por la cual pudieron comunicarse efectivamente en inglés pudo haber sido el resultado de haber compartido largas horas juntos durante este último año, siendo que son amigos, estudian juntos y usan el inglés como medio de comunicación periódicamente. Es evidente que ambos participantes, a lo largo de este tiempo, han logrado acomodar y ajustar exitosamente sus capacidades y habilidades fonológicas tanto productivas como receptivas para poder comunicarse eficiente y efectivamente.

\section{IMPLICANCIAS PEDAGÓGICAS}

En la sección anterior se trataron algunas cuestiones fono-lingüísticas enmarcadas dentro de lo que, hoy en día, se denomina el inglés como lengua internacional. En esta parte del trabajo se abordarán algunos aspectos relacionados con su pedagogía y aplicación (ver Luchini, 2010). El contexto donde se llevó a cabo la experiencia áulica es Discurso Oral II (DOII). DOII es un curso de pronunciación inglesa que se dicta en el segundo año del Profesorado de Inglés de la Universidad Nacional de Mar del Plata, Argentina, luego de que los alumnos han tomado y aprobado Fonética y Fonología Inglesas I y II. El objetivo principal de este curso es, por un lado, equipar a estos futuros profesores de inglés con recursos competitivos que les permitan convertirse en buenos modelos de L2 para sus eventuales alumnos ${ }^{2}$. Asimismo, se espera que los estudiantes de DOII desarrollen una pronunciación aceptable ${ }^{3}$ para

${ }^{2}$ En el caso particular de la adquisición de la pronunciación inglesa para los futuros docentes de inglés, Cruttenden (2001:299) señala: "The foreign teacher of English constitutes a special case. He has the obligation to be as clear as possible about the model towards which he is aiming and to present to his students as near an approximation to that model as he can. Particularly if he is dealing with young pupils, his students will imitate a bad pronunciation as exactly as they will a good one; and if he is using illustrative recorded material, his own pronunciation must not diverge markedly from the pronunciation used in it.

3 Traducción y adaptación del término "acceptability" presentado por Cruttenden (2000). En su libro Gimson's pronunciation of English ( ${ }^{\text {th }}$ Ed.), Cruttenden (2001:299) sugiere el término "HIGH ACCEPTABILITY" como requisito fonético y fonológico indispensable 
poder comunicarse en inglés efectiva y eficientemente no solamente con hablantes nativos de esta lengua sino también con otros hablantes no-nativos, provenientes de otros países.

Los contenidos de DOII se centran, fundamentalmente, en el desarrollo y entrenamiento de los aspectos suprasegmentales de la cadena discursiva inglesa (principalmente, acento, ritmo y entonación) e incluyen, además, algunos de los axiomas de la "Lingua Franca Core" tal cual los presenta Jenkins (2000) en su libro The Phonology of English as an International Language, capítulo 6.

En línea con los objetivos de este curso y para lograr la integración de estos aspectos netamente teóricos con la práctica productiva, en 2008, los alumnos de DOII (de ahora en más denominado Grupo A) completaron una actividad estrictamente centrada en la fonología del inglés como lengua internacional. Para concretar este trabajo, los 21 alumnos fueron divididos en pequeños grupos. A cada grupo se le asignó una de las grabaciones realizadas por los hablantes internacionales (ver sección Instrumentos y procedimientos arriba) para ser transcripta y analizada, siguiendo los lineamientos teóricos presentados por Jenkins (2000) en su "Lingua Franca Core". Una vez completadas estas tareas, cada grupo presentó formalmente sus resultados en clase, ante sus pares. Esta actividad fue desarrollada a lo largo de tres semanas aproximadamente. Hacia la finalización del curso, y luego de haber completado las presentaciones, los alumnos fueron evaluados individualmente mediante un examen oral, administrado por los tres profesores a cargo de DOII. Los resultados de estos exámenes fueron, posteriormente, comparados con los provenientes de otro grupo de alumnos que tomaron el mismo curso en 2009. Este último grupo, denominado $\mathrm{B}$ y conformado por igual número de alumnos que el Grupo A $(n=21)$, si bien cubrió los mismos contenidos teóricos que los participantes del Grupo A, no completó esta actividad.

Por otro lado, al finalizar cada curso (2008 y 2009), ambos grupos (Grupo A y Grupo B) completaron una encuesta evaluativa en donde volcaron sus percepciones acerca de la inclusión del componente que incluyó la enseñanza de la fonología del inglés como lengua internacional. El objetivo de esta comparación fue, justamente, medir el impacto que tuvo la incorporación de esta actividad en la producción oral de los alumnos y evaluar resultados.

A tal fin, se utilizo el método de cegado y se evaluó la diferencia de media aritmética en cada grupo del promedio de los tres profesores. Para el análisis estadístico se utilizó el software SPSS y EPI INFO.

para que todo estudiante de inglés consiga comunicarse efectivamente con hablantes nativos de esta lengua. Este autor inglés define a este término de la siguiente manera: "a form of speech which the native listener may not identify as non-native, which conveys information as readily as would a native's and which arrives at this result through precision in the phonetic realization of phonemes and by confident handling of accentual and intonational patterns." 
En la Tabla 1 debajo se muestra el promedio general de calificaciones de todos los alumnos (Grupos A \& B, $n=42$ alumnos): 5.88 y de sus edades: 23.14. En la Tabla 2, a continuación, se muestran los resultados del Grupo A, en donde se hizo la intervención (actividad) y en la Tabla 3 los resultados del Grupo B (grupo control).

Estadísticos descriptivos

\begin{tabular}{|l|r|r|r|r|r|}
\hline & N & \multicolumn{1}{|c|}{ Mínimo } & \multicolumn{1}{c|}{ Máximo } & \multicolumn{1}{c|}{ Media } & Desv. típ. \\
\hline Teacher_A & 42 & 4 & 8 & 5.83 & 1.124 \\
Teacher_B & 42 & 2 & 9 & 5.81 & 1.642 \\
Teacher_C & 42 & 2 & 8 & 6.02 & 1.388 \\
Edad & 42 & 19 & 48 & 23.14 & 5.749 \\
Prom_teacher & 42 & 2.67 & 8.33 & 5.8889 & 1.23777 \\
N válido (según lista) & 42 & & & & \\
\hline
\end{tabular}

Tabla 1.Resultados de ambos grupos (A \& B)

Estadísticos descriptivos

\begin{tabular}{|l|r|r|r|r|r|}
\hline & \multicolumn{1}{|c|}{ N } & \multicolumn{1}{c|}{ Mínimo } & \multicolumn{1}{c|}{ Máximo } & \multicolumn{1}{c|}{ Media } & Desv. típ. \\
\hline Teacher_A & 21 & 4 & 8 & 6.19 & 1.167 \\
Teacher_B & 21 & 2 & 9 & 6.43 & 1.599 \\
Teacher_C & 21 & 4 & 8 & 6.52 & 1.250 \\
Edad & 21 & 19 & 48 & 23.48 & 6.250 \\
Prom_teacher & 21 & 3.33 & 8.33 & 6.3810 & 1.21237 \\
N válido (según lista) & 21 & & & & \\
\hline
\end{tabular}

Tabla 2. Resultados del Grupo A (intervención)

Estadísticos descriptivos

\begin{tabular}{|l|r|r|r|r|r|}
\hline & \multicolumn{1}{|c|}{ N } & \multicolumn{1}{c|}{ Mínimo } & \multicolumn{1}{c|}{ Máximo } & \multicolumn{1}{c|}{ Media } & Desv. típ. \\
\hline Teacher_A & 21 & 4 & 7 & 5.48 & .981 \\
Teacher_B & 21 & 2 & 9 & 5.19 & 1.470 \\
Teacher_C & 21 & 2 & 8 & 5.52 & 1.365 \\
Edad & 21 & 19 & 41 & 22.81 & 5.335 \\
Prom_teacher & 21 & 2.67 & 8.00 & 5.3968 & 1.07816 \\
N válido (según lista) & 21 & & & & \\
\hline
\end{tabular}

Tabla 3. Resultados del Grupo B (grupo control)

El promedio total de las calificaciones asignadas por los tres profesores para el Grupo A fue de 6.38. El promedio de las calificaciones para el Grupo B fue de 5.39. La diferencia de la media aritmética entre los dos grupos es estadísticamente significativa. La F estadística es alta: 7.92. Esto significa que el efecto de la 
intervención, es decir, la inclusión de la actividad en el Grupo A fue importante. Este resultado no es azaroso sino causal, porque el valor $p$ es 0.007297 . Siendo que ambos grupos fueron homogéneos en términos de la edad de sus participantes, fueron enseñados por los mismos docentes, se utilizaron los mismos materiales de trabajo y se cubrieron los mismos contenidos, evidentemente, la incorporación de la actividad tuvo un fuerte impacto en las producciones de los alumnos del Grupo A. Estos resultados se pueden observar en la Tabla 4 debajo.

\begin{tabular}{|c|c|c|c|}
\hline Grupos & Tamaño & Media & Varianza \\
\hline A & 21 & 6.38 & 1.46 \\
\hline B & 21 & 5.39 & 1.14 \\
\hline \multicolumn{3}{|c|}{ Varianza entre las muestras } & 10.29 \\
\hline \multicolumn{3}{|c|}{ Varianza residual } & 1.30 \\
\hline F estadística & 7.92 \\
\hline Valor de $p$ & 0.007297 \\
\hline
\end{tabular}

Tabla 4. Diferencia de la media aritmética entre grupos

Para validar los resultados de cada docente-evaluador se utilizo el método interevaluación. Al momento de calificar, los evaluadores no fueron informados acerca de quienes habían completado la actividad. Los resultados demostraron que hubo consistencia en la asignación de puntajes por los tres evaluadores. Los tres parecieron haber acordado en bajar considerablemente las calificaciones en el Grupo B. Sin embargo, cada evaluador decidió bajar las calificaciones de manera diferente. El evaluador B (línea verde, en gráfico 1) tuvo una tendencia a sobrevalorar la intervención, con un descenso de $19.3 \%$. El evaluador A (línea azul), por otra parte, desestimó la intervención, marcando un descenso de $11.5 \%$. El evaluador C (línea marrón) fue el más consistente de los tres. Su descenso fue de $15.5 \%$, y coincidió con el descenso indicado por la línea violeta, que muestra el total del promedio de los tres docentes. 


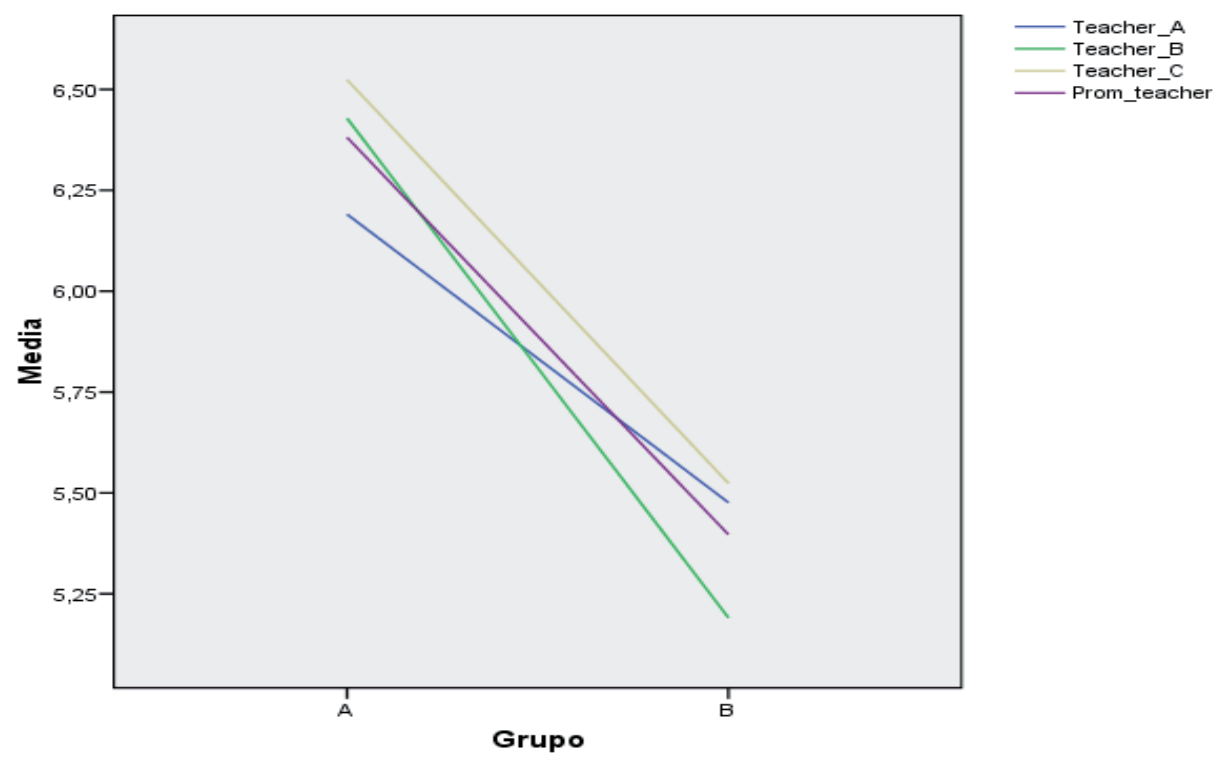

Gráfico 1. Homogeneidad entre los evaluadores

Para el análisis de las encuestas se establecieron categorías emergentes de la literatura vigente sobre sensibilización de la lengua (en inglés, language awareness) y de las mismas respuestas en común, provenientes de los alumnos (Venkatagiri y Levis, 2007; Doughty \& Williams, 1998; Long \& Robinson, 1998; Schmidt; 1990; 1995; White y Ranta, 2002; Lighbown, 2000; Lam, 2009; White y Ranta, 2002; Trofimovich y Kennedy, 2009). Tres categorías fueron exploradas: (i) conocimiento metalingüístico acerca de la pronunciación, (ii) toma de conciencia de un faltante en la lengua (en inglés, "noticing the gap"), y (iii) sensibilización de cómo funciona la lengua para su uso correcto. Estas categorías se presentan debajo en la Tabla 4. Los números hacia la derecha de cada categoría indican los porcentajes obtenidos y el número de alumnos (en paréntesis) en cada grupo que las mencionó en su encuesta.

\begin{tabular}{|l|l|c|}
\hline Categorías & $\begin{array}{l}\text { Grupo A } \\
\text { (intervención) }\end{array}$ & Grupo B (control) \\
\hline Conocimiento metalingüístico & $85 \%$ (18 alumnos) & $28 \%$ (6 alumnos) \\
\hline "Noticing de gap" (input/output) & $57 \%$ (12 alumnos) & $14 \%$ (14 alumnos) \\
\hline $\begin{array}{l}\text { Sensibilización de cómo funciona } \\
\text { la lengua para su uso correcto }\end{array}$ & $76 \%$ (16 alumnos) & $19 \%$ (4 alumnos) \\
\hline
\end{tabular}

Tabla 4. Categorías y porcentajes 
Existe una alta correlación entre los resultados provenientes del análisis estadístico y los comentarios realizados por los alumnos en la encuesta evaluativa. Sus comentarios explican e ilustran con ejemplos concretos algunas de las razones por las cuales los alumnos del Grupo A obtuvieron mejores resultados en sus producciones que los del Grupo B. Este proceso de triangulación de la información contribuyó a validar y fortalecer los resultados obtenidos.

Los alumnos que completaron la actividad (Grupo A) obtuvieron, en general, mejores calificaciones que sus compañeros del Grupo B. Aparentemente, mejor aprendizaje ocurre cuando los estudiantes de una lengua extranjera exploran conscientemente y analizan aspectos teóricos a través de actividades más prácticas.

La creación de una actividad que conecta la teoría con la práctica pareciera ser que permite a los estudiantes comprender acabadamente sus materiales de estudio. El comprender los contenidos de lo que más tarde deberán verbalizar en un examen oral, por ejemplo, les ayuda a organizar su discurso. El descomprimir los contenidos de la temática a desarrollar de sus mentes, aparentemente, los libera de un gran peso cognitivo. En otras palabras, pueden utilizar el tiempo de procesamiento de la información para seleccionar los ítems léxicos y formas fonológicas que luego necesitarán para lograr un discurso cercano al que utiliza un hablante nativo.

Pareciera ser que los enfoques más tradicionales, en donde no se incluye este tipo de actividades, no son lo suficientemente efectivos en generar una toma de consciencia lingüística de los recursos que los alumnos realmente necesitarán para poder comunicarse efectivamente. La adquisición de conocimiento metalingüístico acerca de la pronunciación, por ejemplo, parece ser beneficioso para el desarrollo y entrenamiento de la fonología de una lengua extranjera.

El conocimiento explícito, puesto de manifiesto por medio de esta actividad, pudo haber sido la causa primaria de la mejoría en la producción de los alumnos pertenecientes al Grupo A. En caso de corroborar estos resultados, los profesores de pronunciación deberían, entonces, exponer a sus alumnos a una amplia variedad de acentos ingleses (de hablantes nativos y no nativos) para que los puedan comprender más fácilmente. Parece ser que existe una correlación fuerte entre la comprensibilidad de estos acentos y la precisión fonológica productiva.

\section{DISCUSIÓN Y CONCLUSIÓN FINAL}

El objetivo de este estudio fue identificar aquellas variaciones fonológicas que resultaron ser conducentes a rupturas en el discurso hablado en interacciones entre hablantes de diferentes L2s, provenientes de diversas nacionalidades. De los resultados obtenidos, se puede concluir que las desviaciones en la producción de algunos sonidos, conjuntamente con la incorrecta localización del acento tonal, resultan ser los mayores factores que atentan contra el logro de una mutua inteligibilidad entre L2 hablantes de inglés.

Durante los 54 minutos de grabación, no se registraron casos en los cuales las rupturas en el discurso oral fueran producto de un mal uso de los patrones de 
entonación o contornos entonativos. Esto refuerza la aserción de Jenkins (2000,) en la que afirma que los cambios en altura tonal no suelen afectar significativamente la inteligibilidad entre hablantes no-nativos de inglés de diferentes nacionalidades.

A pesar de que se detectaron algunas inexactitudes a nivel sintáctico y semántico durante las conversaciones grabadas, éstas tampoco obstaculizaron significativamente el fluir de la interacción. Por lo tanto, en este caso particularmente, los resultados muestran que tanto el rol del uso correcto de la entonación, gramática y del vocabulario no son tan indispensables para preservar la inteligibilidad global como sí lo son la correcta localización del acento tonal combinado con algunas desviaciones sonoras como lo anticipa Jenkins (2000) en su LFC ("Lingua Franca Core").

A la luz de estos resultados, y con el objetivo de mejorar la inteligibilidad fonológica en nuestros alumnos, se sugiere, por un lado, brindar vastas oportunidades áulicas a los estudiantes en donde se los exponga a una diversidad considerable de acentos de hablantes nativos y no-nativos de inglés provenientes de diferentes nacionalidades (Luchini, 2005a, 2005b) Por otro lado, se recomienda centrar la enseñanza de la pronunciación en aquellos aspectos o rasgos fonológicos que son vitales para lograr una mutua inteligibilidad (ver marco teórico). Dependerá, entonces, de la voluntad e inquietud de aquellos alumnos en particular que así lo deseen, adquirir una pronunciación que se aproxime a la de los hablantes nativos de inglés.

En cuanto a las implicancias pedagógicas de este tipo de actividades es importante remarcar que aquellos alumnos equipados con estrategias que les permitan notar una cierta sensibilización del lenguaje logran identificar la brecha entre lo que ya han adquirido y lo que necesitan incorporar. Este proceso de toma de consciencia lingüística es conducente a una mejor adquisición del lenguaje.

Con el objetivo de ampliar y expandir los resultados de este estudio, sería interesante continuar investigando esta área con otros participantes y con mayores muestras poblacionales. Esto permitiría a los investigadores en el área establecer nuevas comparaciones y contrastes que, seguramente, aportarán importantes avances al campo de la investigación y didáctica de la fonología del inglés en contextos internacionales.

\section{REFERENCIAS BIBLIOGRÁFICAS}

BROWN, D. (2001). Teaching by principles. An interactive approach to language pedagogy. New York: Longman.

DAUER, R. (2005). The Lingua Franca Core: A New Model for Pronunciation Instruction? TESOL Quarterly, 39/3, 543-550.

DOUGHTY, C. and WILLIAMS, (1998). Focus on Form in Classroom Second language Acquisition. New York: Cambridge University Press.

CRUTTENDEN, A. (2001). Gimson's pronunciation of English (Sixth edition). London: Arnold International Student's Edition. 
HALLIDAY, M. (1985). An introduction to functional grammar. Great Britain: Edward Arnold.

GARCÍA JURADO, M.A. y M. Arenas. (2005). La Fonética del Español. Buenos Aires: Editorial Quórum.

JENKINS, J. 2000. The phonology of English as an international language. Oxford: Oxford University Press.

LAM, Y. (2009). Applying cognitive linguistics to teaching the Spanish prepositions por and para. Language Awareness, 18/1, 2-18.

LEVIS, J. (2005). Changing Contexts and Shifting Paradigms in Pronunciation Teaching. En TESOL Quarterly, 39/3, 369-377.

LIGHBOWN, P. (2000). Classroom SLA research and second language acquisition. Applied linguistics, 21, 431-462.

LONG, M. y ROBINSON, P. (1998). Focus on Form: Theory, research and practice. In Doughty, C. and J. Williams (eds.) Focus on Form in Classroom Second language Acquisition (pp. 15-41). New York: Cambridge University Press.

LUCHINI, P. (2010). Implications for using and teaching English as an International Language: An exploratory study. Presentación realizada en ACLA (Canadian Association of Applied Linguistics), Concordia University, Montreal, Quebec, Canada. 1- 3 junio, 2010.

LUCHINI, P. (2007a). Raising learners' speech awareness through self-assessment and collaborative assessment in the pronunciation class. En IATEFL Speak Out! Newsletter of the IATEFL Pronunciation Special Interest Group. Issue 37.

LUCHINI, P. (2007b). Fonología suprasegmental: una propuesta innovadora. En Fonética y Fonología hoy: Una perspectiva plurilingüe. Universidad Nacional de Córdoba: Comunicarte, Argentina.

LUCHINI, P. y ROSELLÓ, A. (2007). Developing learners' oral communicative language abilities: A collaborative action research project in Argentina. The Journal of Asia TEFL, 4/4, 245-271.

LUCHINI, P. (2006). Suprasegmental Phonology. Handbook for Pronunciation Skill Teaching. Integrating theory with practice. Mar del Plata: Universidad Nacional de Mar del Plata.

LUCHINI, P. (2005a). A New Approach to Teaching Pronunciation: An Exploratory C Case Study. Journal of Asia TEFL: Refereed Journal of the Asian Association of Teachers of English as a Foreign Language, 2/2, 35-62.

LUCHINI, P. (2005b). Integrating a pronunciation component into a Spoken-English course at Shanghai Normal University: A case study. IATEFL Speak Out!Newsletter of the IATEFL Pronunciation Special Interest Group. Issue 32, 14-31.

LUCHINI, P. (2005c). A New Approach to Teaching Pronunciation: An exploratory case study" Journal of Asia TEFL: Refereed Journal of the Asian Association of Teachers of English as a Foreign Language, 2/2, 35-62.

LUCHINI, P. (2004). Designing a pronunciation test for assessing free speech production: an evaluative case study. ATEFL Speak Out! Newsletter of the IATEFL Pronunciation Special Interest Group. Issue 31, 12-24. 
ROGERS, H. (2000). The Sounds of Language. An introduction to phonetics. Harlow: Pearson Education Limited.

SCHMIDT, R. (1990). The role of consciousness in second language learning. Applied linguistics, 11, 129-158.

SCHMIDT, R. (1995). Consciousness and foreign language learning: A tutorial on the role of attention and awareness in learning. In R. Schmidt (ed.) Attention and Awareness in Foreign Language Learning (Tech. Rep. No.9, pp. 1-63). University of Hawaii at Manoa, Second Language Teaching and Curriculum Center.

TROFIMOVICH, P. y KENNEDY, S. (2009). Language awareness and second language pronunciation: A classroom study. Language Awareness, 1-15. (in press)

VENKATAGIRI, H. y LEVIS, J. (2007). Phonological awareness and speech comprehensibility: An exploratory study. Language Awarenes, 16/4, 263277.WHITE, J. y RANTA, L. (2002). Examining the interface between metalinguistic task performance and oral production in second language. Language Awareness, 11/4, 259-290. 


\section{APÉNDICE}

\section{Apéndice A: Tarea (Collaborative Task)}

For this task, you will be asked to work with a partner. You have to imagine that you won a lot of money. You will have 60 seconds to look at some pictures of things that you can do with the money.

Look at the pictures now (60 seconds)...

- picture of a plane (traveling)

- picture of a house on the beach

- picture of a brand-new car

- picture of someone giving out to the poor

- picture of a research laboratory (computers)

You will have to talk to each other and decide which three things you would spend your money on and why. You have about three minutes to talk to each other.

Start talking now...

Please stop talking now. 